\title{
The efficacy of a flipping education program on improving self-management in patients with chronic obstructive pulmonary disease: a randomized controlled trial
}

This article was published in the following Dove Press journal:

International Journal of Chronic Obstructive Pulmonary Disease

\author{
Yi-Ya Chang ${ }^{1,2}$ \\ Yu-Tzu Dai ${ }^{3}$ \\ 'Department of Nursing and Clinical \\ Competency Center, Chang Gung \\ University of Science and Technology, \\ Taoyuan, Republic of China; ${ }^{2}$ Department \\ of Rehabilitation, Chang Gung Memorial \\ Hospital, Taoyuan, Republic of China; \\ ${ }^{3}$ School of Nursing, National Taiwan \\ University, Taipei, Republic of China
}

\begin{abstract}
Purpose: Self-management is widely used among patients with a chronic disease to control their condition. However, the self-management programs are less distinctive for patients with chronic obstructive pulmonary disease (COPD) than those with other chronic diseases. This study examines the efficacy of a flipping education program on improving self-management in patients with COPD.

Patients and methods: A single-blinded, randomized controlled trial was conducted at a medical center in northern Taiwan from January 2015 to May 2016. Sixty participants were randomized to an experimental group and a control group. The self-management program with flipped teaching, customized action plans, and scheduled telephone interviews was implemented in the experimental group for three months. Conventional patient education was implemented in the control group. Disease knowledge, self-efficacy, the patient's activation level, and the impact of COPD were assessed at baseline, 1 month, and 3 months after the intervention. SPSS 22.0 was used for data analysis.

Results: The results showed that the patients who received the flipping education program of self-management had statistically significant improvements in their disease knowledge $(p<0.05)$, self-efficacy $(p<0.01)$, and activation levels $(p<0.01)$ from baseline to the 1 month and 3 months follow-up compared to the control group.

Conclusion: The findings supported that flipped teaching could be applied to patient education in adults and that a nurse case manager can feasibly use this flipping education program of selfmanagement to motivate and support patients with COPD to acquire self-management skills, carry out their action plans, and help them achieve beneficial behaviors in their daily lives.
\end{abstract}

Keywords: patient education, COPD, disease knowledge, self-efficacy, nurse case manager

\section{Introduction}

The morbidity and mortality of chronic obstructive pulmonary disease (COPD) increased due to the factors of smoking, air pollution, and aging populations. By 2030, COPD will be the third leading cause of death globally. ${ }^{1}$ In the United States, the annual medical expenses associated with COPD treatment were US\$50 billion in $2010 .^{2}$ In Taiwan, annual medical spending on COPD is approximately US $\$ 700$ million, ${ }^{3}$ accounting for $4 \%$ of national health insurance expenditures. These statistics underscore the pressing need to address the care of patients with COPD and utilize the medical resources necessary to achieve this objective.
Correspondence: Yi-Ya Chang

Competency Center, Chang Gung University of Science and Technology,

No. 26I, Wenhua Ist Rd., Guishan Dist, Taoyuan City 33303, Republic of China

Tel +886321 I 8999 Ext 3222

Fax +886321I 8866

Email yychang@mail.cgust.edu.tw 
Several interventional studies have associated selfmanagement with improvements in disease knowledge, selfefficacy, reductions in acute exacerbations, admission rates, emergency department (ED) visits, and medical expenses among patients with COPD. ${ }^{4-7}$ Nurses who serve as case managers can effectively help patients with COPD engage in self-management. ${ }^{8} \quad$ However, these patients' selfmanagement practices are less distinctive than those of patients with other chronic diseases. ${ }^{9}$ More evidence on the self-management of patients with COPD is needed.

Nurses teach patients about self-care of diseases typically through one-way teaching. Some high-school teachers in Taiwan have adopted the "flipping-literature class" method to reverse the traditional one-way learning environment by allowing students to self-learn, reflect, and express themselves during instruction. ${ }^{10}$ The method can also be employed to andragogy in this study to prevent the predominantly one-way form of patient education. An instructor of adult students is not only a caretaker, coach, and learning facilitator, but also a counselor. ${ }^{11}$ To build patients' confidence in managing their health, the conceptual framework of the chronic disease self-management program (CDSMP) was used in this study. The CDSMP was developed by researchers at Stanford University and is to empower patients living with a chronic condition. We combined the method of the flipping-literature class, adult learning theory, and the concept of CDSMP to develop a new strategy of the patient education program on self-management for patients with COPD at home. The purpose of this study was to examine the effects of a flipping education program on selfmanagement to improve disease knowledge, self-efficacy, activation level, the impact of COPD, and unexpected medical care in patients with COPD.

\section{Materials and methods}

\section{Design and data collection}

This single-blinded, randomized controlled trial was conducted at a medical center in northern Taiwan from January 2015 to May 2016. The participants in the control group received conventional patient education from two research nurses and those in the experimental group received the flipping education program of self-management from a nurse case manager. A pretest (T0), a post-test performed after completion of intervention 1 month (T1), and a post-test performed after completion of intervention 3 months (T2) were administered during follow-up evaluations through questionnaires completed by two research nurses.

\section{Participants and settings}

Patients diagnosed with COPD were selected from a list of patients who had appointments at the chest outpatient departments of a medical center in northern Taiwan. The eligibility criteria were (1) reading ability, (2) clinical diagnosis of COPD confirmed using the pulmonary function test (FEV1/FVC <0.7) (3) older than 20 years of age, (4) Mandarin-speaking, (5) normal cognition as assessed by the Short Portable Mental State Questionnaire, and (6) a willingness to participate in this study and signed an informed consent form. The exclusion criteria included (1) lung cancer or pulmonary tuberculosis, (2) bronchiectasis, and/or (3) congestive heart failure.

\section{Sample size, allocation, randomization, and blinding}

The sample size was calculated using $G^{*}$ power 3.1 with the mode of $F$ tests MANOVA: repeated measures, between factor; a significance level of 0.05 , power of 0.8 , and with a suggested medium effect size of 0.25 . The total sample size required for this study was calculated to be 22 for each group. Follow-up rates of $50-80 \%$ have been suggested as acceptable. ${ }^{12,13}$ To avoid potential bias, a $20 \%$ attribution rate was calculated, resulting in a total of 27 participants in each group. Our study met the minimum sample size, with 30 participants in each group.

The allocation ratio was 1:1. Excel's RAND function was used to generate a set of six random numbers and reordered the group $\mathrm{ABABAB}$ by repeatedly ranking numbers 10 times. Each number in the conventional patient education group or the flipping education program of selfmanagement was kept separate in a sealed envelope. When the baseline data were completed, the research nurses allocated the participants into groups according to the random ranking number.

A single-blinded, randomized controlled trial was designed. All of the participants in this study were told that the nurses would teach them how to take care of themselves before informed consent was obtained. The participants in both groups received interventions and were blinded as to whether they were in the control group or the experimental group. Two research nurses were responsible for collecting the questionnaires and administering the conventional patient education to the participants in the control group in a classroom at the medical center. 


\section{Ethical considerations}

The Research Ethics Committee at National Taiwan University Hospital approved this study (201112134RIC). This study was conducted in accordance with the Declaration of Helsinki. The protocol has been registered on the National Institutes of Health website (NCT01931267). Informed consent was obtained from all of the participants. Prior to the study, all of the participants were told that they were free to withdraw at any time and for any reason.

\section{Interventions}

MSRAA flipping education program in the experimental group

A three-month motivating, self-learning, reflecting, action plan verbalizing, and action taking (MSRAA) flipping education program of self-management was proposed and administered to the experimental group. The patient-education approach used in the MSRAA flipping education program of self-management was adapted from the method of "flipping literature class"10 and differed from conventional patient education, with the case manager encouraging patients to self-learn the self-management booklet and discussing with them the goals of their chosen self-management action plans. The self-management booklet ${ }^{14}$ was developed by the Health Promotion Administration.

The MSRAA flipping education program of selfmanagement were simply strategies used by a nurse manager to promote self-management program. The first author of this study served as the case manager who has received $15 \mathrm{hrs}$ of training from instructors who had certificated from Stanford's CDSMP. In the first stage of motivating, the nurse case manager motivated the participants to self-learn about COPD selfmanagement from the self-management booklet through motivation interviewing. In the second stage of selflearning, the participants self-learned before discussing with the nurse case manager. Also, the nurse case manager made a phone call to make sure the participants have learned the booklet and to make an appointment with them to discuss. In the third stage of reflecting and action plan verbalizing, the nurse case manager asked the participants to reflect why they suffered from COPD and discussed their self-management with them for 1-1.5 hrs to help them create custom action plans (such as pursed-lip breathing, smoking cessation, adopting environmental control measures, preventing respiratory tract infections, taking medications regularly, and exercising regularly). In the final stage of action-taking, the case manager conducted scheduled telephone interviews (10-15 mins/time) with the participants during the second, third, fourth, eighth, and twelfth weeks of the program to motivate them and track their progress on their chosen action plans, record their difficulty in executing their action plans, and discuss how to solve their difficulty with them.

\section{Conventional patient education in the control group} The control participants received conventional patient education from the research nurses using patient education handouts provided by the medical center. Compared to the experimental group, the research nurses taught the patients about self-care of diseases typically through oneway instruction without asking them to reflect and discussing their disease with them. The control group participants did not have clear action plan goals or scheduled telephone interviews from a case manager to motivate them.

\section{Pilot phase}

Four participants with a diagnosis of COPD were invited to the pilot study prior to the trial. From the pilot study, we found that different levels of Likert scales were not easy to select for the participants, and they recommended that it was better to have paper-based questionnaires during the face-to-face interviews. After the pilot study, paper-based questionnaires with a font size of 24 and reading glasses were prepared for the participants, and a different number of charts were used to show the varying levels of Likert scales at the trial commencement.

\section{Outcome measures}

The intervention outcomes were determined using data collected through the administration of the Chronic Obstructive Pulmonary Disease Knowledge Questionnaire (COPD-Q), the Pulmonary Rehabilitation Adapted Index of Self-Efficacy (PRAISE), the Patient Activation Measure (PAM), and the COPD Assessment Test (CAT). The authors of the COPD-Q, PRAISE, and PAM gave informed consent for use of the scales prior to the data collection.

The COPD-Q, a questionnaire developed to evaluate COPD knowledge, has acceptable internal consistency (Cronbach's $\alpha=0.72)$ and high reliability $(\gamma=0.9) .{ }^{15}$ The COPD-Q is comprised of 13 true or false questions. If one question is answered correctly, the respondent receives 1 point; the total score ranges from 0 to 13 . Higher COPD-Q scores indicate a deeper understanding 
of COPD. The Chinese version of the COPD-Q was translated by the authors and the translation accuracy was confirmed via back-translation; the Kuder-Richardson reliability was 0.55 in this study.

The PRAISE is comprised of 15 items. Higher PRAISE scores indicate greater self-efficacy. The instrument has high internal consistency (Cronbach's $\alpha=$ 0.95). ${ }^{16}$ The Chinese version of the PRAISE was translated by Chan. ${ }^{17}$ Cronbach's $\alpha$ was 0.906 for the Chinese version of the PRAISE in this study.

The PAM was developed to determine the activation level of patients and examine the knowledge, skills, and confidence needed to manage an illness. ${ }^{18}$ Those with higher activation levels have a lower degree of fatalism about their health. The measure has both a 22- and 13-item version. The 13-item PAM was administered in this study with converted total scores ranging from 0 to 100 . Higher PAM scores indicate greater patient activation. Hsu translated the English version of the PAM into Chinese and found good internal consistency in the 22-item Chinese version (Cronbach's $\alpha=0.88$ ). ${ }^{19}$ Cronbach's $\alpha$ was 0.905 for the Chinese version of the PAM in the 13-item version in this study.

The CAT measures the impact of COPD on a patient's life and physical and mental health. It is comprised of eight questions measured on a 5-point scale, with the total score ranging from 0 to 40 . Higher CAT scores indicate higher impact of COPD. The CAT has a Cronbach's $\alpha$ of $0.88 .^{20}$ Cronbach's $\alpha$ of the Chinese version was 0.641 in this study.

The researcher recorded the frequency of acute exacerbation and unexpected medical care of the participants at the first, third, and sixth months following the intervention. In addition, the confidence level was used to measure the subjects' confidence in practicing their action plans in the experimental group. Those with higher confidence levels indicate their belief in their own ability to be successful in their action plans. Unexpected medical care including readmission, ED visits, or unexpected outpatient visits for respiratory symptoms were collected six months after the intervention.

\section{Statistical methods}

Statistical analyses were performed using SPSS 22.0. Analyses were intention to treat and included the participants lost to follow-up. Chi-square tests, t-tests, and the repeated measures analysis generalized estimating equation (GEE) were used in this study. The significance level was set at $\alpha=0.05$.

\section{Results}

\section{Recruitment and participant flow}

The study flowchart (Figure 1) shows the trial recruitment according to CONSORT guidelines. A total of 1,123 patients with a diagnosis of COPD were screened in this study. The most frequent reason for not meeting the inclusion criteria was that patients refused to participate in this study $(n=464)$. Among the 464 patients that refused to participate in this study, $225(48.50 \%)$ patients were in the mild stage, 128 (27.58\%) patients in the moderate stage, and 111 (23.92\%) patients in the severe stage of severity in airflow obstruction. Sixty patients consented to participate and took the pretest. Ultimately, 27 participants remained in the experimental group and 25 in the control group at the three-month follow up. The attrition rate was $13.3 \%$.

\section{Participants' baseline characteristics}

Table 1 shows the baseline characteristics. No significant differences were found in the baseline characteristics between the groups. The participants were an average age of 72.03 years. Most were male $(\mathrm{n}=55 ; 91.7 \%)$, mild stage of severity in airflow obstruction $(\mathrm{n}=35 ; 58.3 \%)$, COPD group A $(\mathrm{n}=31$; $51.7 \%$ ), and had quit smoking ( $\mathrm{n}=42 ; 70 \%)$. Table 2 presents the outcomes of the analysis in different instruments before the intervention (T0). Results showed no significant differences between two groups in COPD-Q (knowledge of COPD), PAM (patient activation level), and CAT (the impact of COPD) from the two sample $t$-test analysis. Also, it showed a significant difference in PRAISE (self-efficacy).

\section{The impact of the MSRAA flipping education program on outcomes}

Table 3 presents the outcomes of the GEE analysis and the effect size in different instruments at different time. The outcome of GEE analysis showed an interaction between group and time. According to the GEE statistical results, the COPD-Q scores, the PRAISE scores, and the PAM scores of the experimental group were statistically significant and increased compared to the control group at both $\mathrm{T} 1$ and $\mathrm{T} 2$. It revealed that the CAT scores of the experimental group were statistically significant and decreased compared to the control group at $\mathrm{T} 1(P=0.011)$, but no significant decrease compared to the control group at $\mathrm{T} 2(P=0.180)$.

The CAT does not have any subscales, and the limited size of the study sample made it impossible to perform a factor analysis. Three categories were established through 


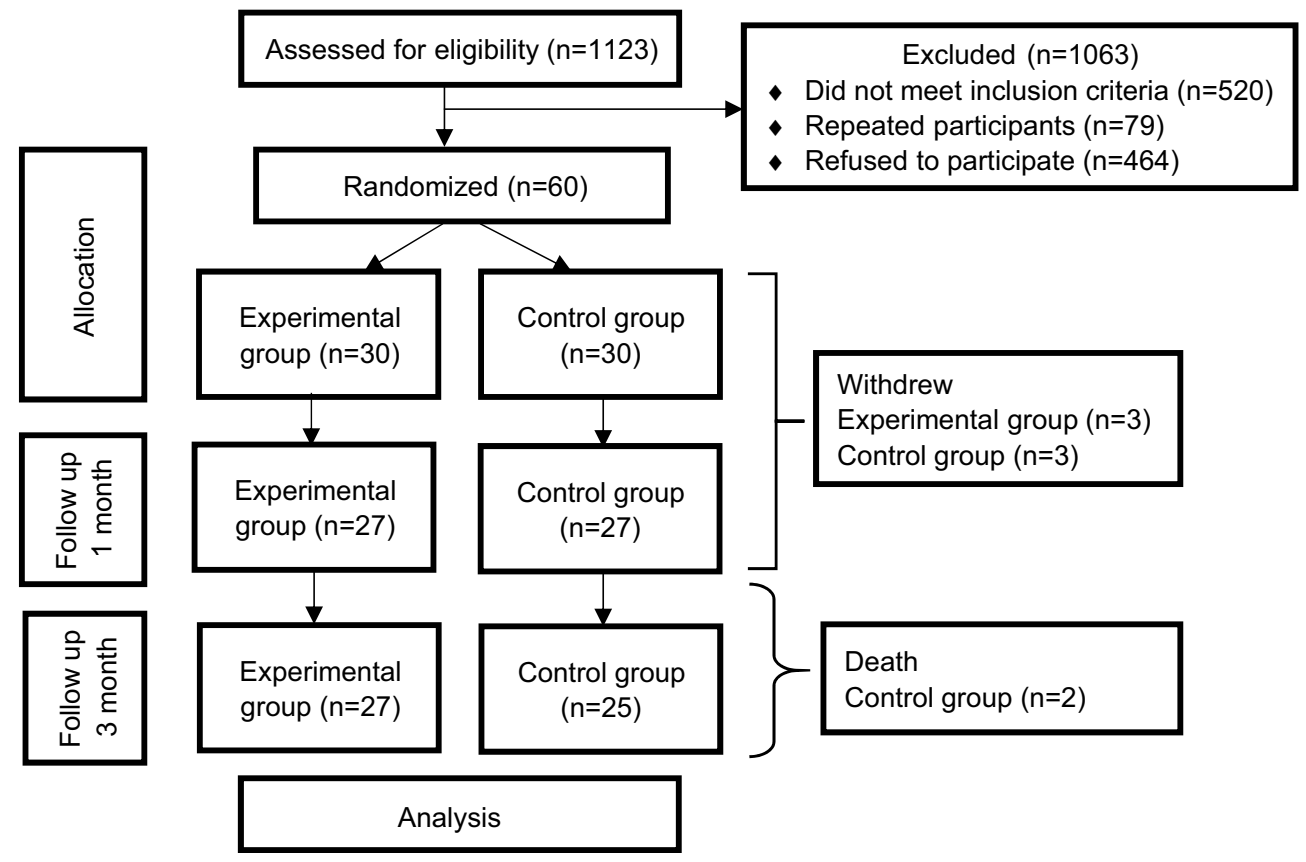

Figure I Study flowchart.

the meaning of each question: symptoms (Items 1-3), physical activity (Items 4-6), and impact (Items 7 and 8). Table 4 shows the GEE analysis of the impact of COPD. The GEE analysis revealed that the score for the symptoms category in the experimental group was significantly lower than that of the control group at both $\mathrm{T} 1$ and $\mathrm{T} 2$.

The experimental group achieved a large effect size at T1 (Cohen's d =0.904) and a medium effect size at T2 (Cohen's $\mathrm{d}=0.525)$ in patient's knowledge and a medium effect size at $\mathrm{T} 1$ (Cohen's $\mathrm{d}=0.483$ ) and T2 (Cohen's $\mathrm{d}=0.403$ ) in patient's activation level. In addition, a medium effect size was shown in self-efficacy (Cohen's $d=0.441$ ) and the impact of COPD (Cohen's $d=0.426$ ) at $\mathrm{T} 1$ and a small effect size was shown in self-efficacy (Cohen's $d=0.138$ ) and the impact of COPD (Cohen's d $=0.172$ ) at T2.

\section{Unexpected medical care}

Table 5 shows that no significant differences in unexpected medical care between the groups from the $t$-test analysis. Fourteen of the participants reported being treated at an ED and hospitalized because of respiratory problems during the 6-month tracking period following the intervention. In particular, 11 participants reported experiencing acute exacerbation during this period, with an incidence of $21.2 \%$. An analysis of the characteristics of the 11 participants found that those who visited the ED because of acute exacerbations were in risk groups $\mathrm{C}(\mathrm{n}=2)$ and $\mathrm{D}(\mathrm{n}=2)$. Most of the participants who had sought medical care continued smoking (22.2\%) and had moderately severe COPD (35.3\%); notably, two were in COPD group $\mathrm{C}$, and another two were in group D. According to the combined COPD Assessment from the Global Initiative for Chronic Obstructive Lung Disease, patients in different groups have a different level of risk and symptoms. Patients in group A belong low risk and less symptoms, patients in group B belong low risk and more symptoms, patients in group $\mathrm{C}$ belong high risk and less symptoms, and patients in group D belong high risk and more symptoms. ${ }^{21}$

\section{Execution of self-management action plans}

Of the 30 participants in the experimental group, 3 withdrew because of time constraints. The remaining 27 completed the MSRAA flipping education program of selfmanagement. Pursed-lip breathing was the most adopted strategy $(n=27 ; 100 \%)$, followed by regular exercise $(n=25 ; 92.59 \%)$, regular taking of medications $(n=12$; $44.44 \%)$, prevention of respiratory tract infections $(n=10$; $37.03 \%)$, smoking cessation $(n=10 ; 37.03 \%)$, and the adoption of environmental control measures $(n=3$; $11.11 \%)$. The confidence level of the experimental group concerning the execution of their action plans ranged between 5 and 10 (the highest level being 10), with a mean level of $7.85(\mathrm{sd}=1.375)$. The records of scheduled 
Table I Baseline characteristics of participants $(\mathrm{N}=60)$

\begin{tabular}{|c|c|c|c|c|c|c|c|}
\hline \multirow[t]{2}{*}{ Characteristics } & \multicolumn{2}{|c|}{$\begin{array}{l}\text { All participants } \\
(n=60)\end{array}$} & \multicolumn{2}{|c|}{$\begin{array}{l}\text { Experimental group } \\
(n=30)\end{array}$} & \multicolumn{2}{|c|}{$\begin{array}{l}\text { Control group } \\
(n=30)\end{array}$} & \multirow[t]{2}{*}{ P-value } \\
\hline & $\mathbf{n}$ & $\%$ & $\mathbf{n}$ & $\%$ & $\mathbf{n}$ & $\%$ & \\
\hline Gender & & & & & & & 0.643 \\
\hline Male & 55 & $91.7 \%$ & 27 & $90 \%$ & 28 & $93 \%$ & \\
\hline Female & 5 & $8.3 \%$ & 3 & $10 \%$ & 2 & $7 \%$ & \\
\hline Age & 72.03 & 11.14 & 70.5 & 11.26 & 73.55 & 10.99 & 0.293 \\
\hline Severity of airflow obstruction & & & & & & & 0.418 \\
\hline Mild & 35 & $58.3 \%$ & 15 & $50.0 \%$ & 25 & $66.7 \%$ & \\
\hline Moderate & 17 & $28.3 \%$ & 10 & $33.3 \%$ & 7 & $23.3 \%$ & \\
\hline Severe & 8 & $13.3 \%$ & 5 & $16.7 \%$ & 3 & $10.0 \%$ & \\
\hline COPD groups ${ }^{\mathrm{a}}$ & & & & & & & 0.267 \\
\hline$A$ & 31 & $51.7 \%$ & 15 & $50.0 \%$ & 25 & $66.7 \%$ & \\
\hline B & 25 & $41.7 \%$ & 11 & $36.7 \%$ & 14 & $46.7 \%$ & \\
\hline C & 2 & $3.3 \%$ & 2 & $6.7 \%$ & 0 & $0.0 \%$ & \\
\hline D & 2 & $3.3 \%$ & 2 & $6.7 \%$ & 0 & $0.0 \%$ & \\
\hline Employment status & & & & & & & 0.375 \\
\hline Full-time job & 10 & $16.7 \%$ & 7 & $23.3 \%$ & 3 & $10.0 \%$ & \\
\hline Part-time job & 6 & $10.0 \%$ & 3 & $10.0 \%$ & 3 & $10.0 \%$ & \\
\hline Retired & 44 & $73.3 \%$ & 20 & $66.7 \%$ & 24 & $80.0 \%$ & \\
\hline Smoking history & & & & & & & 0.195 \\
\hline Never & 9 & $15.0 \%$ & 2 & $6.7 \%$ & 7 & $23.3 \%$ & \\
\hline Already quit & 42 & $70.0 \%$ & 23 & $76.7 \%$ & 19 & $63.3 \%$ & \\
\hline Still smoking & 9 & $15.0 \%$ & 5 & $16.7 \%$ & 4 & $13.3 \%$ & \\
\hline Realize diagnosed with COPD & & & & & & & 0.197 \\
\hline Yes & 12 & $20 \%$ & 8 & $26.7 \%$ & 4 & $13.3 \%$ & \\
\hline No & 48 & $80 \%$ & 22 & $73.3 \%$ & 26 & $86.7 \%$ & \\
\hline Vaccination against influenza & & & & & & & 0.260 \\
\hline Yes & 42 & $70 \%$ & 19 & $63.3 \%$ & 23 & $76.7 \%$ & \\
\hline No & 18 & $30 \%$ & 11 & $36.7 \%$ & 7 & $23.3 \%$ & \\
\hline Vaccination against pneumococcal conjugate & & & & & & & 0.542 \\
\hline Yes & 14 & $23.3 \%$ & 6 & $20 \%$ & 8 & $26.7 \%$ & \\
\hline No & 46 & $76.7 \%$ & 24 & $80 \%$ & 22 & $73.3 \%$ & \\
\hline
\end{tabular}

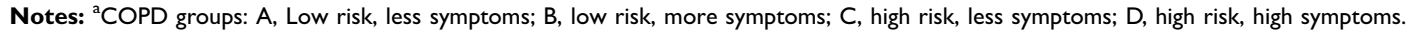

Table 2 Baseline analysis in different instruments $(\mathrm{N}=60)$

\begin{tabular}{|l|l|l|l|l|}
\hline Outcome measure & $\begin{array}{l}\text { Experimental group }(\mathbf{n = 3 0}) \\
\text { Mean } \pm \text { SD }\end{array}$ & $\begin{array}{l}\text { Control } \\
\text { group }(\mathbf{n = 3 0}) \\
\text { Mean } \pm \text { SD }\end{array}$ & t-test \\
\hline Disease knowledge (COPD-Q) & $7.57 \pm 2.49$ & $8.07 \pm 1.72$ & -1.139 & 0.259 \\
Self-efficacy (PRAISE) & $43.50 \pm 5.73$ & $47.30 \pm 7.86$ & -2.144 & $0.036 *$ \\
Patient activation level (PAM) & $59.64 \pm 14.38$ & $65.59 \pm 16.20$ & -1.504 & 0.138 \\
Impact of COPD (CAT) & $9.93 \pm 6.57$ & $8.60 \pm 4.76$ & 0.900 & 0.372 \\
\hline
\end{tabular}

Note: $* P<0.05$. 
Table 3 Generalized estimating equation analysis of longitudinal outcomes and effect size in different instruments $(N=60)$

\begin{tabular}{|c|c|c|c|c|c|c|}
\hline $\begin{array}{l}\text { Outcome measure } \\
\text { (instrument) }\end{array}$ & Variable & Estimate & SE & $\begin{array}{l}\text { Wald chi- } \\
\text { square }\end{array}$ & $P$-value & $\begin{array}{l}\text { Effect } \\
\text { size }\end{array}$ \\
\hline Disease knowledge (COPD-Q) & $\begin{array}{l}\text { Intercept } \\
\text { Experimental group }^{\mathrm{a}} \\
\mathrm{T} 2^{\mathrm{b}} \\
\mathrm{T} 1^{\mathrm{b}} \\
\text { Experimental group* }^{*} \\
\mathrm{~T}^{\mathrm{c}} \\
\text { Experimental group* }^{*} \\
\mathrm{TI}^{\mathrm{c}}\end{array}$ & $\begin{array}{l}7.267 \\
-0.633 \\
0.853 \\
0.326 \\
1.661 \\
\\
2.300\end{array}$ & $\begin{array}{l}0.3331 \\
0.5465 \\
0.5083 \\
0.4349 \\
0.7904 \\
0.6245\end{array}$ & $\begin{array}{l}475.874 \\
1.343 \\
2.819 \\
0.562 \\
4.419 \\
13.563\end{array}$ & $\begin{array}{l}<0.00 I^{* * *} \\
0.247 \\
0.093 \\
0.454 \\
0.036 * \\
<0.00 \text { I*** }\end{array}$ & $\begin{array}{l}0.525 \\
0.904\end{array}$ \\
\hline Self-efficacy (PRAISE) & $\begin{array}{l}\text { Intercept } \\
\text { Experimental group } \\
\mathrm{T}^{\mathrm{b}} \\
\mathrm{T} 1^{\mathrm{b}} \\
\text { Experimental group* } \\
\mathrm{T}^{\mathrm{c}} \\
\text { Experimental group* }^{*} \\
\mathrm{TI}^{\mathrm{c}}\end{array}$ & $\begin{array}{l}47.300 \\
-3.800 \\
1.540 \\
-1.448 \\
4.812 \\
6.763\end{array}$ & $\begin{array}{l}1.4065 \\
1.7422 \\
1.2141 \\
1.4894 \\
1.7132 \\
1.8682\end{array}$ & $\begin{array}{l}1131.023 \\
4.757 \\
1.609 \\
0.945 \\
7.889 \\
13.105\end{array}$ & $\begin{array}{l}<0.00 I^{* * *} \\
0.029 * \\
0.205 \\
0.331 \\
0.005^{* *} \\
<0.00 I^{* * *}\end{array}$ & 0.138 \\
\hline Patient activation level (PAM) & $\begin{array}{l}\text { Intercept } \\
\text { Experimental group }^{\mathrm{a}} \\
\mathrm{T} 2^{\mathrm{b}} \\
\mathrm{T} 1^{\mathrm{b}} \\
\text { Experimental group* }^{*} \\
\mathrm{~T}^{\mathrm{c}} \\
\text { Experimental group* }^{*} \\
\mathrm{TI}^{\mathrm{c}}\end{array}$ & $\begin{array}{l}65.593 \\
-5.950 \\
3.64 \mid \\
0.034 \\
12.158 \\
13.887\end{array}$ & $\begin{array}{l}2.9075 \\
3.8885 \\
3.1225 \\
3.3237 \\
4.2178 \\
\\
4.5567\end{array}$ & $\begin{array}{l}508.964 \\
2.341 \\
1.360 \\
0.000 \\
8.309 \\
9.288\end{array}$ & $\begin{array}{l}<0.00 I^{* * *} \\
0.126 \\
0.244 \\
0.992 \\
0.004^{* *} \\
0.002^{* *}\end{array}$ & 0.403 \\
\hline Impact of COPD (CAT) & $\begin{array}{l}\text { Intercept } \\
\text { Experimental group }^{\mathrm{a}} \\
\mathrm{T} 2^{\mathrm{b}} \\
\mathrm{Tl}^{\mathrm{b}} \\
\text { Experimental group* }^{\mathrm{b}} \\
\mathrm{T}^{\mathrm{c}} \\
\text { Experimental group* } \\
\mathrm{Tl}^{\mathrm{c}}\end{array}$ & $\begin{array}{l}8.600 \\
1.333 \\
0.120 \\
2.437 \\
-2.239 \\
-3.926\end{array}$ & $\begin{array}{l}0.8545 \\
1.4564 \\
1.236 \\
1.0803 \\
1.6715 \\
\\
1.5527\end{array}$ & $\begin{array}{l}101.284 \\
0.838 \\
0.009 \\
5.089 \\
1.794 \\
6.393\end{array}$ & $\begin{array}{l}<0.00 I^{* * * *} \\
0.360 \\
0.923 \\
0.024^{*} \\
0.180 \\
0.011^{*}\end{array}$ & $\begin{array}{l}0.172 \\
0.426\end{array}$ \\
\hline
\end{tabular}

Notes: $* P<0.05, * * P<0.01$, and $* * * P<0.001$. ${ }^{a}$ Reference group: control group, ${ }^{b}$ reference group: T0 (baseline), ${ }^{c}$ reference group: control group $* T 0$ (T0=baseline, $\mathrm{TI}=\mathrm{I}$ month after intervention, $\mathrm{T} 2=3$ months after intervention).

telephone interviews from patients in the experimental group were analyzed by content analysis. Four factors that affected the self-management behaviors in patients with COPD were environment, time, physical discomfort, and the difficulty of action plan.

\section{Discussion}

\section{Outcomes of the MSRAA flipping education program}

The purpose of this study was to evaluate the efficacy of a flipping education program on self-management in patients with COPD. The results suggested that the
MSRAA flipping education program of selfmanagement helped to improve the participants' knowledge of COPD, self-efficacy, activation levels, and the impact of COPD.

The growth of the patients' knowledge was the most significant. The experimental group that with a large effect size at T1 (Cohen's $d=0.904$ ) and a medium effect size at T2 (Cohen's $d=0.525$ ) achieved a significantly higher mean post-test score for patient's knowledge $(P<0.001$ at $\mathrm{T} 1 ; P=0.036$ at $\mathrm{T} 2)$ than did the control group. It showed that knowledge is the easiest to improve to patients with COPD. 
Table 4 Generalized estimating equation analysis of longitudinal outcomes in the impact of COPD (N=60)

\begin{tabular}{|c|c|c|c|c|c|}
\hline Category & Variable & Estimate & SE & Wald chi-square & $P$-value \\
\hline \multirow[t]{6}{*}{ Items I 3 symptoms } & Intercept & 4.033 & 0.477 & 71.431 & $<0.001$ \\
\hline & Experimental group ${ }^{a}$ & 0.567 & 0.730 & 0.602 & 0.438 \\
\hline & $\mathrm{T} 2^{\mathrm{b}}$ & 0.497 & 0.530 & 0.879 & 0.348 \\
\hline & $\mathrm{TI}^{\mathrm{b}}$ & 1.039 & 0.536 & 3.755 & 0.053 \\
\hline & Experimental group ${ }^{*} \mathrm{~T} 2^{\mathrm{c}}$ & -1.794 & 0.7626 & 5.535 & $0.019 *$ \\
\hline & Experimental group ${ }^{*} \mathrm{TI}^{\mathrm{c}}$ & -1.855 & 0.7215 & 6.606 & $0.010 *$ \\
\hline \multirow[t]{6}{*}{ Items $4 \sim 6$ activity } & Intercept & 1.733 & 0.3494 & 24.612 & $<0.001$ \\
\hline & Experimental group ${ }^{a}$ & 0.767 & 0.6086 & 1.587 & 0.208 \\
\hline & $\mathrm{T} 2^{\mathrm{b}}$ & 0.028 & 0.4432 & 0.004 & 0.95 \\
\hline & $\mathrm{TI}^{\mathrm{b}}$ & 0.738 & 0.4305 & 2.939 & 0.086 \\
\hline & Experimental group ${ }^{*} \mathrm{~T} 2^{\mathrm{c}}$ & -0.979 & 0.7575 & $1.67 \mid$ & 0.196 \\
\hline & Experimental group ${ }^{*} \mathrm{TI}^{\mathrm{C}}$ & -1.763 & 0.7384 & 5.704 & $0.017^{*}$ \\
\hline \multirow[t]{6}{*}{ Items 7 8 impact } & Intercept & 2.833 & 0.4057 & 48.763 & $<0.001$ \\
\hline & Experimental group ${ }^{a}$ & 0.000 & 0.6415 & 0.000 & 1.000 \\
\hline & $\mathrm{T} 2^{\mathrm{b}}$ & -0.406 & 0.5587 & 0.529 & 0.467 \\
\hline & $\mathrm{TI}^{\mathrm{b}}$ & 0.693 & 0.4417 & 2.465 & 0.116 \\
\hline & Experimental group ${ }^{*} \mathrm{~T} 2^{\mathrm{c}}$ & 0.507 & 0.6465 & 0.614 & 0.433 \\
\hline & Experimental group ${ }^{*} \mathrm{TI}^{\mathrm{c}}$ & -0.371 & 0.5580 & 0.442 & 0.506 \\
\hline
\end{tabular}

Notes: $* P<0.05$. ${ }^{a}$ Reference group: control group, ${ }^{b}$ reference group: $\mathrm{T} 0$ (baseline), ${ }^{c}$ reference group: control group $* \mathrm{~T} 0$ (T0=baseline, $\mathrm{Tl}=\mathrm{I}$ month after intervention, $\mathrm{T} 2=3$ months after intervention).

Table 5 Comparisons in unexpected medical care for respiratory problems $(\mathrm{N}=52)$

\begin{tabular}{|l|l|l|l|l|l|l|}
\hline \multirow{2}{*}{ Outcome measure } & \multicolumn{2}{l|}{ Experimental group (n=27) } & \multicolumn{2}{l|}{ Control group (n=25) } & \multirow{2}{*}{ t-test } \\
\cline { 2 - 6 } & Frequency & Mean \pm SD & Frequency & Mean \pm SD & \\
\hline Unexpected outpatient visit & 12 & $0.48 \pm 0.64$ & 11 & $0.44 \pm 0.58$ & 0.243 & 0.809 \\
Emergency visit & 8 & $0.30 \pm 0.61$ & 7 & $0.28 \pm 0.54$ & 0.102 & 0.919 \\
Hospitalization & 2 & $0.11 \pm 0.42$ & 5 & $0.20 \pm 0.41$ & -0.769 & 0.445 \\
Total & 22 & $0.88 \pm 1.19$ & 23 & $0.92 \pm 0.97$ & -0.102 & 0.919 \\
\hline
\end{tabular}

Before the intervention, the average self-efficacy scores in the experimental group and control group were $43.50(\mathrm{sd}=5.73)$ and $47.30(\mathrm{sd}=7.86)$, which were significantly different $(P=0.036)$. The participants in the control group had better self-efficacy than the experimental group. The experimental group achieved a significantly higher post-test score for self-efficacy than did the control group ( $P=0.000$ at $\mathrm{T} 1 ; P=0.005$ at $\mathrm{T} 2$ ), indicating improvement in the confidence of patients with COPD who participated in the MSRAA flipping education program of self-management. This finding agreed with a research, ${ }^{5}$ which found that self-management interventions can enhance patients' self-efficacy.

The experimental group with a medium effect size (Cohen's $d=0.483$ at T1; Cohen's $d=0.403$ at T2) achieved a significantly higher mean post-test score of patient activation levels $(P=0.002$ at $\mathrm{T} 1 ; P=0.004$ at T2) than did the control group, suggesting improvements in the self-management knowledge, skills, and confidence of the patients with COPD who participated in the MSRAA flipping education program of self-management.

Before the intervention, the average CAT scores of the experimental group were higher (with higher impact of COPD) than the control group. After the intervention, the experimental group had lower CAT scores (with lower impact of COPD), significantly different than the control group at T1. This indicated that the MSRAA flipping education program of self-management can improve the impact of COPD in patients with COPD. In addition, the results of the analysis indicated no significant differences in the score of the overall impact of COPD between the experimental and control groups but significant differences 
in the scores of symptoms (Items 1-3) at both T1 and T2. Thus, the MSRAA flipping education program of selfmanagement facilitated the alleviation of COPD symptoms, and this finding corresponded with a previous study that self-management improves breathing symptoms in patients with COPD. ${ }^{22}$

No significant difference in the frequency of unexpected medical care was observed between the groups, possibly because most of the participants in this study belonged to COPD groups A (low risk and fewer symptoms; 31 participants) and B (low risk and more symptoms; 25 participants) and were therefore at low risk of acute exacerbation. Our study showed that patients with COPD in groups C and $\mathrm{D}$ experienced acute exacerbations more readily than those in risk groups A and B. Similarly, some studies ${ }^{23,24}$ found that, over a 1-year tracking period, patients with high COPD severity and poor pulmonary function were prone to acute exacerbations. Thus, individuals in COPD high-risk groups should receive more instruction on identifying and responding to acute exacerbations.

\section{Factors affecting the outcomes of the MSRAA flipping education program}

A systematic literature review ${ }^{25}$ of the effective components of the self-management of COPD and congestive heart failure found high heterogeneity in the selfmanagement outcomes between both diseases, which made it difficult to identify the components of selfmanagement that effectively improved the quality of life. A single influencing factor was not derived from the study because the self-management of daily life is characteristically multidimensional and involves multiple components.

Previous studies found that patient education is critical to the use of self-management by individuals with COPD and improves patients' knowledge of the disease. ${ }^{26,27}$ All subjects in this study were adults, who require instruction in a manner that differs from traditional approaches. The case manager plays a crucial role in adult learning in this study. The nurse case manager worked as a booster to encourage the participants to learn about selfmanagement by themselves for preparatory work, and then the nurse case manager discussed with them for $1-1.5 \mathrm{hrs}$. It was more effective compared to the $2-10-\mathrm{hr}$ individualized and group education programs in previous studies on COPD self-management. ${ }^{5,8,28}$ It showed that self-learning was feasible for the patients with COPD in this study.
Individuals with COPD may be willing to strive for wellness in their daily lives and act accordingly. ${ }^{29}$ Selfmanagement interventions should be developed in a manner that can strengthen the intrinsic motivation of these individuals to engage in self-management and adopt healthy behaviors associated with this practice. ${ }^{30}$ In this study, the case manager encouraged the participants to articulate their expectations about disease improvement - thereby strengthening their intrinsic motivation to engage in selfmanagement and their desirable goals for action plans. Because the participants customized their self-management action plans, they committed closely to the plans and were encouraged by the case manager during telephone interviews to work toward their respective self-management objectives. This finding corresponded with a previous study that customized self-management plans are crucial to deepening the involvement of individuals with COPD. ${ }^{31}$

The subjects in the current study were asked to implement customized action plans that were within their capabilities. Lorig et al demonstrated that when patients have a confidence level of $\geq 7$, they can successfully fulfill the goals of their self-management action plans. ${ }^{32}$ The mean of the confidence level in this study was 7.85, suggesting that the subjects were adequately confident about achieving the objectives of their own action plans and improved noticeably in patient activation levels. Also, more than $90 \%$ of the subjects in the experimental group choose to practice pursed-lip breathing and regular exercise in their daily lives. This finding corresponded with a previous study $^{33}$ that patients with COPD were directed towards the prevention, control, and management of the physical consequences of COPD for self-care.

The majority of the subjects in this study did not realize that they had been diagnosed with COPD $(n=48$; $60 \%$ ). Jonsdottir et al reported a similar finding, suggesting that some of their subjects $(24 \%)$ did not realize they had been diagnosed with COPD. ${ }^{28}$ Researchers found that $15.9 \%$ of their subjects who had developed hypertension were not aware of their affliction with this chronic illness. ${ }^{34}$ From the result of this study and previous studies, ${ }^{28,34}$ more people are unaware of their affliction with COPD than those with hypertension. The level of COPD knowledge may affect the completion of action plans adopted and the number of unexpected outpatient visits. ${ }^{35}$ A number of 225 patients in the mild stage of severity in airflow obstruction refused to participate in this study. The reasons could be that patients' life were less affected by the disease symptoms and so their illness 
perceptions and their willingness to participate in this study were lower. Researchers recommend that action needs to be taken to reach out to young people with COPD in its early stages. ${ }^{28}$ When people acquire knowledge of a disease, they normally change their attitudes and behaviors toward it. Thus, health care professionals should provide detailed information regarding COPD and the stages thereof to enable patients with this disease to adopt self-management practices as early as possible to delay its progression.

In the present study, $70 \%$ of the subjects had been vaccinated against influenza, compared to $49.4 \%, 62.7 \%$, and $76.2 \%$ reported in previous studies. ${ }^{35-37}$ However, far fewer subjects had received a pneumococcal conjugate vaccine $(n=14 ; 23.3 \%)$ than had those enrolled in a study $(65.5 \%)^{38}$ The fact that more subjects in the present study had been vaccinated against influenza than pneumococcus was probably due to the restrictions placed on publicly funded vaccinations by government policy in Taiwan; senior citizens (65 years or older) are eligible to receive an influenza vaccine at public expense, while only those aged 75 years or older are eligible to receive a free pneumococcal conjugate vaccine. ${ }^{39}$ Patients with COPD receive both influenza and pneumococcal conjugate vaccines are reported to pay fewer visits to EDs and outpatient clinics. ${ }^{36}$ Preventing the development of pneumonia is paramount for individuals with COPD; the government needs to fund the vaccination of high-risk patients against pneumococcus. Future studies on COPD self-management should compare the number of subjects vaccinated against influenza and pneumococcus before and after self-management interventions are administered.

\section{Liminations}

This study had three limitations. The small sample size in this study is a limitation. The tracking period spanned only six months. However, because acute exacerbations are more likely to occur in winter, a period of the winter season should be included for the observation of every subject to correctly elucidate the occurrence of acute exacerbation. Future researchers should track individuals with COPD for up to one year to investigate changes in their disease status over this period.

This study recruited patients with COPD only from a medical center in northern Taiwan. Thus, the findings may not apply to COPD individuals with different characteristics. Future studies should investigate larger samples of patients with COPD recruited from multiple health care institutions or geographical locations to yield findings with greater objectivity and extrapolability.

\section{Conclusion}

The results of this study supported that the MSRAA flipping education program of self-management can help patients with COPD improve their knowledge, selfefficacy, activation level, and the impact of COPD and to engage in beneficial behaviors in their daily lives. The MSRAA flipping education program of self-management is feasible for use by nurses, offering an alternative to the conventional patient education method, and can motivate and support patients with COPD to acquire selfmanagement knowledge and skills and implement selfmanagement in their daily lives.

\section{Data sharing statement}

The datasets generated and analyzed during the current study are available from the corresponding author on reasonable request for 10 years since this study conduct date. Any other study-related documents will be provided upon request as well.

\section{Acknowledgments}

All authors express special thanks to all participants who shared their valuable experiences to this study. This study was supported by the Ministry of Science and Technology Department (101-2314-B-002-050-MY 3, 2012-2016) in Taiwan. This paper/The abstract of this paper was presented at the Asian Conference on Psychology \& the Behavioral Sciences 2017 as a poster presentation with interim findings. The poster's abstract was not published.

\section{Author contributions}

The conception and design of this study came from Yi-Ya Chang. In addition, Yi-Ya Chang performed data analysis and wrote the draft of the manuscript. Yu-Tzu Dai contributed to data analysis as well as the drafting of the manuscript. Both the authors have approved the final manuscript and agree to be accountable for all aspects of the work.

\section{Disclosure}

The authors report no conflicts of interest in this work. 


\section{References}

1. World Health Organization [homepage on the Internet]. Chronic obstructive pulmonary disease (COPD). Fact. Available from: https:// www.who.int/respiratory/copd/en/. Accessed January 14, 2019.

2. Guarascio AJ, Ray SM, Finch CK, Self TH. The clinical and economic burden of chronic obstructive pulmonary disease in the USA. Clinicoecon Outcomes Res. 2013;5:235-245. doi:10.2147/CEOR.S34321

3. Ministry of Health and Welfare. Smoking resulted in COPD that spent 20 billion of health insurance [homepage on the Internet]. [updated November 15, 2011]. Available from: https://tw.news.yahoo.com $/ \%$ E5\%90\%B8\%E8\%8F\%B8\%E5\%B0\%8E\%E8\%87\%B4copd-\%E5\% B $\%$ B $4 \%$ E $8 \% 8$ A $\% 1 \%$ E $\% 81 \%$ A5\% E4\%BF\%9D\%Е $\% \%$ B2\% BB200\%E5\%84\%84-075300420.html. Accessed August 15, 2015.

4. Bourbeau J, Saad N, Joubert A, et al. Making collaborative self-management successful in COPD patients with high disease burden. Respir Med. 2013;107(7):1061-1065. doi:10.1016/j. rmed.2013.03.003

5. Fan VS, Gaziano JM, Lew R, et al. A comprehensive care management program to prevent chronic obstructive pulmonary disease hospitalizations: a randomized, controlled trial. Ann Intern Med. 2012;156:673-683. doi:10.7326/0003-4819-156-10-20120515000003

6. Sánchez-Nieto JM, Andújar-Espinosa R, Bernabeu-Mora R, et al. Efficacy of a self-management plan in exacerbations for patients with advanced COPD. Int $J$ Chron Obstruct Pulmon Dis. 2016;11:1939-1947.

7. Aboumatar H, Naqibuddin M, Chung S, et al. Effect of a program combining transitional care and long-term self-management support on outcomes of hospitalized patients with chronic obstructive pulmonary disease: a randomized clinical trial. Jama. 2018;320 (22):2335-2343. doi:10.1001/jama.2018.17933

8. Benzo R, Vickers K, Ernst D, Tucker S, McEvoy C, Lorig K. Development and feasibility of a self-management intervention for chronic obstructive pulmonary disease delivered with motivational interviewing strategies. J Cardiopulm Rehabil Prev. 2013;33 (2):113-123. doi:10.1097/HCR.0b013e318284ec67

9. Disler RT, Gallagher RD, Davidson PM. Factors influencing self-management in chronic obstructive pulmonary disease: an integrative review. Int J Nurs Stud. 2012;49(2):230-242. doi:10.1016/j. ijnurstu.2011.11.005

10. Chang HC. Flipped teaching: the cultivation of self-learning ability and new education of library. NCKU Lib J. 2016;25:1-7.

11. Wallace S, Gravells J. Mentoring in Future Education. Exeter: Learning Matters; 2007.

12. Babbie E. Survey Research Methods. Belmont. CA: Wadsworth Publishing; 1973.

13. Kristman V, Manno M, Cote P. Loss to follow-up in cohort studies: how much is too much? Eur J Epidemiol. 2004;19(8):751-760.

14. Administration HP. Self-management in COPD. 2006.

15. Maples P, Franks A, Ray S, Stevens AB, Wallace LS. Development and validation of a low-literacy Chronic obstructive pulmonary disease knowledge questionnaire (COPD-Q). Patient Educ Couns. 2010;81(1):19-22. doi:10.1016/j.pec.2009.11.020

16. Vincent E, Sewell L, Wagg K, Deacon S, Williams J, Singh S. Measuring a change in self-efficacy following pulmonary rehabilitation: an evaluation of the PRAISE tool. Chest. 2011;140 (6):1534-1539. doi:10.1378/chest.10-2649

17. Chan H-Y, Dai Y-T, Hou IC. Evaluation of a tablet-based instruction of breathing technique in patients with COPD. Int J Med Inform. 2016;94:263-270. doi:10.1016/j.ijmedinf.2016.06.018

18. Hibbard JH, Stockard J, Mahoney ER, Tusler M. Development of the patient activation measure (PAM): conceptualizing and measuring activation in patients and consumers. Health Serv Res. 2004;39(4 Pt 1):1005-1026. doi:10.1111/j.1475-6773.2004.00269.x
19. Hsu YW A Study on Developing of Patient Activation Measurement and It's Relationship with Patient Behavior Focus on Diabetes Patients [Master thesis]. National Taiwan University; 2005.

20. Jones PW, Harding G, Berry P, Wiklund I, Chen WH, Kline Leidy N. Development and first validation of the COPD assessment test. Eur Respir J. 2009;34(3):648-654. doi:10.1183/09031 936.00102509

21. Global Initative for Chronic Obstructive Ling Disease. Global strategy for the diagnosis, management, and prevention of COPD 2018 report. Available from: https://goldcopd.org/wp-content/uploads/ 2017/11/GOLD-2018-v6.0-FINAL-revised-20-Nov_WMS.pdf. Accessed November 1, 2018.

22. Johnson-Warrington V, Rees K, Gelder C, Morgan MD, Singh SJ. Can a supported self-management program for COPD upon hospital discharge reduce readmissions? A randomized controlled trial. Int J Chron Obstruct Pulmon Dis. 2016;11:1161-1169. doi:10.2147/COPD.S91253

23. Kim JK, Lee SH, Lee BH, et al. Factors associated with exacerbation in mild- to-moderate COPD patients. Int J Chron Obstruct Pulmon Dis. 2016;11:1327-1333. doi:10.2147/COPD.S105583

24. Margüello MS, Garrastazu R, Ruiz-Nuñez M, et al. Independent effect of prior exacerbation frequency and disease severity on the risk of future exacerbations of COPD: a retrospective cohort study. NPJ Prim Care Respir Med. 2016;26:16046. doi:10.1038/npjpcrm.2016.46

25. Jonkman NH, Westland $\mathrm{H}$, Trappenburg JC, et al. Do self-management interventions in COPD patients work and which patients benefit most? An individual patient data meta-analysis. Int J Chron Obstruct Pulmon Dis. 2016;11:2063-2074. doi:10.2147/ COPD.S107884

26. Cannon D, Buys N, Sriram KB, Sharma S, Morris N, Sun J. The effects of chronic obstructive pulmonary disease self-management interventions on improvement of quality of life in COPD patients: a meta-analysis. Respir Med. 2016;121:81-90. doi:10.1016/j.rmed.2016.11.005

27. Paneroni M, Clini E, Crisafulli E, et al. Feasibility and effectiveness of an educational program in Italian COPD patients undergoing rehabilitation. Respir Care. 2013;58(2):327-333. doi:10.4187/respcare.01697

28. Jonsdottir H, Amundadottir OR, Gudmundsson G, et al. Effectiveness of a partnership-based self-management programme for patients with mild and moderate chronic obstructive pulmonary disease: a pragmatic randomized controlled trial. $J$ Adv Nurs. 2015;71 (11):2634-26492616p. doi:10.1111/jan.12728

29. Chang YY, Dai YT, Chien NH, Chan HY. The lived experiences of people with chronic obstructive pulmonary disease: a phenomenological study. J Nurs Scholarsh. 2016;48(5):1-6. doi:10.1111/jnu.12230

30. Bourbeau J, Lavoie KL, Sedeno M. Comprehensive self-management strategies. Semin Respir Crit Care Med. 2015;36(4):630-638. doi:10.1055/s-0035-1556059

31. Korpershoek Y, Bos-Touwen ID, de Man-van Ginkel JM, Lammers JW, Schuurmans MJ, Trappenburg J. Determinants of activation for self-management in patients with COPD. Int $J$ Chron Obstruct Pulmon Dis. 2016;11:1757-1766.

32. Lorig K, Holman H, Sobel D, Laurent D, Gonzalez V, Minor M. Living a Healthy Life with Chronic Conditions. CO: Bull Publishing Company; 2006.

33. Clari M, Matarese M, Ivziku D, Marinis M. Self-care of people with chronic obstructive pulmonary disease: a meta-synthesis. Patient. 2017;10(4):407-427. doi:10.1007/s40271-017-0218-z

34. Paulose-Ram R, Gu Q, Kit B. Characteristics of U.S. Adults with hypertension who are unaware of their hypertension, 2011-2014. NCHS Data Brief. 2017;278:1-8.

35. Choi JY, Chung H-IC, Han G. Patient outcomes according to COPD action plan adherence. $J$ Clin Nurs. 2014;23(5/6):883-891. doi:10.1111/jocn.12293

36. Garrastazu R, García-Rivero JL, Ruiz M, et al. Prevalence of influenza vaccination in chronic obstructive pulmonary disease patients and impact on the risk of severe exacerbations. Archivos De Bronconeumologi'a. 2016;52(2):88-95. doi:10.1016/j.arbres.2015.09.001 
37. Santos-Sancho JM, Jimenez-Trujillo I, Hernández-Barrera V, et al. Influenza vaccination coverage and uptake predictors among Spanish adults suffering COPD. Hum Vaccin Immunother. 2012;8 (7):938-945. doi:10.4161/hv.20204

38. Carreño-Ibáñez LV, Esteban-Vasallo MD, Domínguez-Berjón MF, et al. Coverage of and factors associated with pneumococcal vaccination in chronic obstructive pulmonary disease. Int J Tuberc Lung Dis. 2015;19(6):735-741. doi:10.5588/ijtld.14.0480
39. Centers for Disease Control. Taiwan: recommendations of vaccination in adults and elderly [homepage on the Internet] [cited April 25, 2016]. Available from: http://www.cdc.gov.tw/list.aspx?treeid= D78DE698C2E70A89\&nowtreeid=660E95AF8C5B4AB5. Accessed April 25, 2016.

\section{Publish your work in this journal}

The International Journal of COPD is an international, peer-reviewed journal of therapeutics and pharmacology focusing on concise rapid reporting of clinical studies and reviews in COPD. Special focus is given to the pathophysiological processes underlying the disease, intervention programs, patient focused education, and self management

Submit your manuscript here: https://www.dovepress.com/international-journal-of-chronic-obstructive-pulmonary-disease-journal protocols. This journal is indexed on PubMed Central, MedLine and CAS. The manuscript management system is completely online and includes a very quick and fair peer-review system, which is all easy to use. Visit http://www.dovepress.com/testimonials.php to read real quotes from published authors. 